Research Article

\title{
Unearthing the Barriers and Facilitators to the Initiation of Midwifery Programme in India-An Exploratory Case Study
}

\author{
Priya', Manju Chhugani ${ }^{2}$, Merlin Mary James ${ }^{3}$
}

${ }^{1}$ M.Sc. Nursing Student, ${ }^{2}$ Dean, ${ }^{3}$ Ex-faculty, School of Nursing Sciences \& Allied Health, Rufaida College of Nursing, Jamia Hamdard, Block-D, Hamdard Nagar, New Delhi, India.

DOI: https://doi.org/10.24321/2455.9318.202010

\section{I $\quad \mathbf{N} \quad \mathbf{F} \quad \mathbf{O}$}

\section{Corresponding Author:}

Priya, School of Nursing Sciences \& Allied Health, Rufaida College of Nursing, Jamia Hamdard, Block-D, Hamdard Nagar, New Delhi, India.

E-mail Id:

ps359659@gmail.com

Orcid Id:

https://orcid.org/0000-0002-2204-5838

How to cite this article:

Priya, Chhugani M, James MM. Unearthing the Barriers and Facilitators to the Initiation of Midwifery Programme in India-An Exploratory Case Study. Int J Nurs Midwif Res 2020; 7(1): 53-61.

Date of Submission: 2020-04-17

Date of Acceptance: 2020-05-21

\section{$\begin{array}{llllllll}\mathbf{A} & \mathbf{B} & \mathbf{S} & \mathbf{T} & \mathbf{R} & \mathbf{A} & \mathbf{C} & \mathbf{T}\end{array}$}

Objective: Currently the maternal mortality in India is 130 per 100,000 live births (SRS) and nearly 1000 women now die of pregnancy-related complications each month. Midwifery might not be a comprehensive answer to India's maternity mess, but it is an important and necessary part of the answer, the present study is intended to explore the barriers and facilitators to the initiation of midwifery programme.

Materials and Method: A qualitative research approach using exploratory case study design was used to explore the perceived barriers and facilitators to the initiation of midwifery programme among key stakeholders of midwifery including midwifery educators, obstetricians, nurses, administrators, NGO personnel and policy makers. Data were collected from 21 key stakeholders of midwifery using individual indepth interview with in-depth interview guide using audio recorder. The data was analyzed using thematic analysis.

Result: Based on the interview, five themes emerged as perceived barriers to the initiation of midwifery programme and six themes as perceived facilitators to the initiation of midwifery programme. The barriers were categorized as individual, workplace, societal, educational and policy level and facilitators were sensitization among the society on midwifery, prospective about profession, educational organization, research evidences, enablers or enabling activities from higher authorities and support from other countries and professional activities. The results revealed that the major barrier was workplace barrier and major facilitator was enablers from higher authority, as drawn from the QDA miner software $14.56 \%$ and $11.45 \%$, respectively. Conclusion: Our results supports that it is vital to sensitize the community to have a natural birthing experience and instill a desire in them to receive a continuum of care by midwives.

Keywords: Midwifery Programme, Key stakeholder, Barriers, Facilitators, Initiation, India 


\section{Introduction}

Each second, approximately 4.3 births occur throughout the world. Some births bring happiness, while some other bring sadness, many are wanted, however many are unwanted, some are born into poverty and a few into wealth, whatever be the circumstances; those who have given birth know and feel its importance of having a child. Those who observe the birth, whether they are a midwife, birth attendants, partner, friends, family or stranger, know they have been part of a process that ensure our species' continuing existence and exemplifies human essence. ${ }^{1}$

India is the second most populous country of the world that is stood at 1.21 billion comprising of 623.72 million (51.54\%) males and 586.46 million (48.46\%) females (By Government of India Ministry of Health and Family Welfare December 2011 census) and has fast changing socialpolitical demographic patterns that have been drawing global attention in recent years. ${ }^{2}$ According to WHO 2018, every day, approximately 830 women die from preventable causes related to pregnancy and childbirth, which are 536000 maternal deaths globally out of which 117000 (22\%) occur in India. ${ }^{2}$

One of the solutions, experts agree, is to bring in midwives- it would lower the caesarean birth rates, reduce unnecessary risk of women and their babies, and improve maternal and infant health overall, as global evidence shows. ${ }^{3}$

India's first midwifery course, a pilot programme was launched by state government of Telangana in October 2017 in the district of Karimnagar. As Telangana had the highest rates of caesarean births in the country, with $74.9 \%$ in the private sector and $40.3 \%$ in public sector, according to National Family Health Survey (2015-2016). Globally, $10-15 \%$ is considered the "ideal rate", as per the WHO. ${ }^{3}$

Evidences indicates that when midwives are educated to international standards, it could avert more than $80 \%$ of all maternal deaths, still births and neonatal deaths. Achieving this impact also requires that midwives are licensed, regulated, fully integrated into health systems and working in inter-professional teams. ${ }^{4}$

According to WHO, factors that prevent women from receiving or seeking care during pregnancy and child birth are: - poverty, distance, lack of information, inadequate services and cultural practices. In India failure in the health care system, lack of man power and apathy towards patient care can be the factors. ${ }^{5}$

The present study is intended to explore the barriers and facilitators to the initiation of midwifery programme. It will help the policy makers to have a better understanding to the identified barriers and facilitators, for addressing those barriers and maximizing the facilitators. A case study had been conducted in Manitoba, on barriers and facilitators related to implementation of regulated midwifery, themes are identified under barriers and future strategies for sustaining the midwifery profession focused on ensuring some key barriers. ${ }^{6}$

\section{Statement of the Problem}

An Exploratory Study to Assess the Barriers and Facilitators to the Initiation of the Midwifery Programme among Key Stakeholders of Midwifery in New Delhi, India.

\section{Research Questions}

1. What are the barriers to the initiation of midwifery programme among key stakeholders of midwifery in New Delhi, India?

2. What are the facilitators to the initiation of midwifery programme among key stakeholders of midwifery in New Delhi, India?

\section{Materials and Methods \\ Study Design}

A purely qualitative research using case study- explorative design was used to explore the perceived barriers and perceived facilitators to the initiation of midwifery programme. The study utilized the purposive sampling technique. This involves selecting participants on the basis of their characteristics, roles or experiences in order to shed light to arrange of issues relevant to research question. The samples were key stakeholders of midwifery in New Delhi, India. The midwifery educators, administrators, policy makers, NGO personnel, obstetricians and nurses (RN, RM) working in the field of midwifery (Labour room, Antenatal ward, Postnatal ward, Family planning or Maternity operation theater) towards ensuring quality maternal and child health care. Interviewing guide was used to make sure that participant had covered all the issues as per the research questions, but often they addressed spontaneously. The researcher spent 1 month (October 2019) for collecting data.

\section{Data Collection}

Data were collected in New Delhi, India during the month of October 2019. Population in the present study was key stakeholders of midwifery including Midwifery Educators, Obstetricians, Nurses, Administrators, NGO personals and Policy Makers in New Delhi, India (Table 1). With the help of advisors, the study participants were carefully recruited and represented, keeping in mind the researcher's intention for the study.

\section{Ethical Considerations}

Formal ethical permission obtained from The Jamia Hamdard Institutional Ethical Committee (JHIEC). 
Table I.Key Stakeholders

\begin{tabular}{|c|c|c|c|}
\hline $\begin{array}{c}\text { S. } \\
\text { No. }\end{array}$ & $\begin{array}{c}\text { Participant's } \\
\text { characteristics }\end{array}$ & Frequency & Percentage \\
\hline 1. & Job titles & & \\
\hline a) & Midwifery educators & 04 & $19.04 \%$ \\
\hline b) & Administrators & 03 & $14.28 \%$ \\
\hline c) & Nurses & 02 & $9.52 \%$ \\
\hline d) & Obstetricians & 03 & $14.28 \%$ \\
\hline f) & NGO Personnel & 05 & $23.80 \%$ \\
\hline g) & Policy maker & 04 & $19.04 \%$ \\
\hline
\end{tabular}

An in-depth interview schedule was prepared. The interview scheduled developed was subjected to validation from experts in the field of midwifery and nursing and the research committee. Suggestions were incorporated and amendments were made in the in-depth interview schedule before administering to the key- stakeholders. A pilot study was done on 2 participants; after getting informed consent. Respondents reported that interviews were not biased and they were 'comfortable' with the questions.

In order to have full access to their perceived barriers and facilitators, the researcher conducted 21 individual in-depth interviews to collect data. Data were collected using audiotape recorder which recorded the in-depth interviews, all of which were conducted by the researcher. Data were collected by interactive interview using the interview guide after taking written consent.

\section{Data Analysis}

The analysis of data began during the interview and continued as they were transcribed to ascertain sufficient data to represent the various aspects under study. After the transcripts of the interviews were produced, the researcher had two major tasks. The first is translation (through editing and reorganization). The task called for utmost patience and persistence from the part of the researcher. The second is to provide insightful interpretation of perception of the key stakeholders regarding the barriers and facilitators to the initiation of midwifery programme with in a knowledge to action framework. A thematic analysis was done. Respondent's number had been denoted as ' $R$ ' beneath the verbatim.

"Midwifery Programme" is a profession embedded in it and for the actual initiation of this programme, there are some potential and perceived barriers or obstacles.

Based on the interview five themes emerged as barriers to the initiation of midwifery programme, which are as follows:

\section{Theme I: Individual Barriers}

Individual barriers largely depend upon how a stakeholder perceives midwifery as a profession in India and what entails a professional midwife to deliver safe, skilled and sensitive care to the women. Under this theme there were two sub themes.

\section{Subtheme I: Lack of Personal Attributes among Nurses}

Greatest barrier to the initiation of midwifery programme in India are the midwives themselves, because of their lack of confidence, competence, commitment and willingness to take up the daunting challenge of defining and recognizing midwifery as a profession.

Nursing is a profession; all the students who undergo this training receive a qualification of Diploma in General Nursing and Midwifery or Bachelors in Nursing, which yields them a Registered Nurse and Registered Midwife number, however in the course of time, focus has been given largely on nursing and midwifery has been sidetracked and not given much attention.

"At least nurses realize that they are nurses and have started fighting for that. All these nurses are midwives too and are not fighting for that. Nurses call themselves a nurse and not as midwives, often I think that is also something which act as a barrier"(R18).

"I think pre-service education is structured in a way where even though nurses get a license to be a nurse and a midwife ( $R N, R M)$, but the degree that they receive is B.Sc.(Hons) Nursing or B.Sc. Nursing so, midwifery doesn't appear there anywhere" (R18).

\section{Subtheme 2: Lack of adaptability of proficiency among Nurses}

Many of the stakeholders shared that, they also have observed lack of professional standards among nurses which has created a huge impact in nursing profession as a whole. Nurses have become so dependent on others and also lack the professional commitment, which has resulted in their loss of identity in the healthcare team, as a key member.

"This is very sad to say, students are pursuing masters in midwifery and they are not able to do perform per abdominal examination, how can then someone expect that they will conduct normal deliveries independently" (R15).

\section{Theme: 2 Workplace Barriers}

The actual setting of practice for the midwives, are not favorable, which eventually acts as hindrance to the initiation of midwifery programme.

\section{Subtheme: Workplace Environment}

There is a famous saying that "Nurses are the heart of the hospital and Doctors are the brain of the hospital", although both of them are the core members of the healthcare team, 
yet it has been perceived that doctors are a hindrance for this programme as perceived:

The dominance of doctors at workplace leaves the mothers with no choice for opting the mode of delivery, and more so, the nurses are merely following the doctor's orders, rather than exercising their own skills:

"The doctors are dominating the labour room and they do not allow the nurses to do their functions. It completely depends upon the choice of the doctors to take the decision not the patient's choice; likewise, nurses are also restricted by the doctor's decision" (R04).

"If we look at health care as a team where every member of the team has a different and unique role to play, it is important to respect each other and give the space for each other to exercise their respective roles" (R18).

'Birth' is the evolution in nature, but in this materialistic world people are seeing all the profession for the sake of money making only so, this the concern that people are taking this as a business which eventually acts as a barrier to the programme. Doctors will oppose this as they will be concerned as they will lose their patients to midwifery care.

"Doctors will again start hindering, as they will lose their patients because midwives will start taking care of mother during antenatal period, intranatal period and postnatal period. Doctors won't allow nurses to take up the role as they will think that the patient will shift to the midwives" (R11).

\section{Subtheme: Work Culture}

Working style of the nurses can be a hindrance.

"Everything regarding delivery and all relevant decisions are dealt by the doctors only, and nurses are just for the documentation, which consumes their maximum time" (R11).

As there are overcrowding at work place which leads to burn out among nurses which affects the care they are giving to their patients, there is a disparity in the working style of doctors and nurses, nurses are meant to work with the client to make them ventilate their feelings and emotions but now it is not possible for nurses because there are lots of patients for them to look after and are not able to give detailed attention to an individual patient.

"Burn out is happening among nurses and midwives and eventually they are losing out compassion" (R18).

\section{Sub theme: Recruitment Issues}

The issues that are due to the unclear job description or legal protection at work place, have emerged from the improper recruitment of the staff. Creation of the posts is one of them, and for candidates applying for this programme, there must be some kind of security and protection at work place which is still a barrier in India, as it takes too much of efforts of so many people and from so many authorities.

"Even though there will be midwife-led care unit, the admission will happen under the obstetrician, so it is imperative that there is legal protection for nurses at workplace" (R08).

Nurses are much involved in the nursing as compared to the midwifery. They are involved but the fact is that we are not having many jobs in midwifery area, or those who are working in the midwifery area are not practicing it, which is largely because of the absence of job description.

\section{Subtheme: Lack of Career Development Plan}

Progression is the development at the work place, and the pathway for the progression for a midwife is not clearly laid out, and what is that one thing that will attract candidates to enroll into this programme should be defined. There is no legal framework and scope of practice which again stands like a hindrance to the programme.

"The fact is that we don't have the legal framework for midwifery within the country as of now, scope of practice to midwifery has not been defined and that's really creating barriers to the launch of this programme" (R06).

\section{Subtheme: Inadequate Facilities}

Facilities are the resources or place where they are going to work as an independent practitioner. Although, we are promising to give mother, quality cares which is impossible with inadequate resources.

"Although we have achieved a decent percentage of institutional deliveries after the implementation of Janani Suraksha Yojana, but we have not been able provide to quality care to the mothers because of lack of adequate facilities. There are no enough beds for mothers, and one bed space is occupied by two mothers, so it is a matter of concern as to whether our country is prepared for this programme with its existing resources in the hospitals" (R18).

\section{Theme 3: Societal Barriers}

Barriers created by the community towards the midwifery programme, as Nurses have broken up the trust of the public by their own activities by not giving them scientific reasons for the things they do and not practicing their job and this causes the lack of trust on the nurses.

\section{Subtheme: Discrepancy in Opinion}

There is a difference in the opinion of the public towards the midwife, as for some the term midwife is totally unrecognized. The difference in their perceptions about this profession is because midwifery is not in practice, even nurses themselves feels a stigma towards midwifery as they don't like themselves to called are 'Midwives'. For 
them nursing is a more prestigious profession as compared to midwifery.

"Why nurses are not involving with midwifery is because status is not given to them, they consider the midwives' status is lower than the nurses and hence, most of the nurses do not want to do work in midwifery area, and are afraid and ashamed to call themselves as a midwife" (R04).

\section{Theme 4: Educational Barrier}

Education in nursing has become a source of endorsement. In recent times, there have been a lot of mushrooming of nursing schools and colleges and some of them are established solely for the purpose of money making. They give degree to the students, without them being actually exposed to the clinical settings.

\section{Subtheme: Lack of Educational Resources}

As of now we don't have anything which is like midwife-led care unit, so establishing them is also one of the concerns because of the lack of basic requirement like a functional hospital for the candidates to practice their skills.

"The infrastructure or financial resources for the setting up of the midwifery led care unit needs to be sorted out first so that it caters to the training needs of the newly enrolled candidates" (R06).

"The educational institutes should have well trained faculty and a good clinical area for practice. Many times, the institutes start up with the programme with a business intention, and students take admission and they finish with their course without any hands-on practice. Somehow the examinations are conducted and the students are made to pass without actual clinical experience" (R17).

"I feel that as a student it is important to have hands on practice and the teacher's role is vital in that. I don't know how many midwifery teachers have conducted deliveries and their competencies" (R20).

"Syllabus is good; competency is there; only practice is not there" (R21).

\section{Subtheme: Lack of Clinical Exposure}

The clinical areas are shared by the nursing as well as medical student which again makes nurses less competent. There is a lack of human touch in nurses, because they have been stressing more on finishing their assignments during their training period, rather than actually being with the mother during labour.

"In some hospitals the same labour room has been used for medical students and nursing students and medical students are given more chance for conducting delivery but nursing students just watch them do and their casebooks are filled without actually conducting deliveries" (R04).

\section{Theme 5: Barrier at Policy Level}

To initiate any programme, there is always a collaboration of many key stakeholders, both at centre and state level.

\section{Subtheme: Employee Non-friendly Management}

As some of the key stakeholders addressed that there is a weak political will and support in our country to initiate any programme and its take so much of time in our country for any implementation. Another issue is that there are no nurses at policy level, who will speak for the nurses. The committee for implementing such programme comprises of representatives from almost all other field, except from nursing and midwifery.

"One barrier is that we have very less nurses at the policy level, who will speak up for nurses, no one is going to speak up, because it is an only the nurse who can speak for a nurse" (R01).

"The key barrier which I feel is, to bring all stakeholders together in one platform with a similar opinion" (R06).

\section{Facilitators}

If the things which act as a barrier to the programme are corrected, then to a certain extent it may facilitate this programme. Based on the interview five themes emerged as facilitators to the initiation of midwifery programme, which are as follows:

\section{Theme I: Sensitization among the Society on Midwifery}

Nurses are actively working in our country, however midwives have lost their visibility because they are less active in our community and people are not aware about who a midwife is and what they are doing, so it sometimes becomes difficult to trust them easily since for the last several decades, people are going to the doctors, so it is important and the need of the hour to sensitize the community about who a midwife is and what is their scope of practice.

\section{Subtheme: Supportive Mechanisms}

To sensitize the community, we need some mechanism or instruments which will help in orienting the people about the profession of midwifery. Many places in rural areas, the normal deliveries are still conducted by the nurses, but the thing is that nobody knows them that they are the midwives. Propaganda can also be a facilitator for this programme, to spread the positive image of the midwife, to make the people get aware about the new concepts and this awareness and recognition will give further more space to the midwifes to be in the community.

"If we go to the rural area, we will not find the doctors doing all the delivery but some are done by nurses and 
they are doing it very nicely, it's just that we have to give them 'a name', and make them realize of whatever they are doing is important and equip them with little bit of extra skills" (R01).

Our media always shows the bad image of childbirth in India, so it is important to sensitize the country with the good image of midwifery.

There is a dire need for awareness regarding the image of the midwife and their work not only in the general population but also among the health care professionals.

"Midwifes will be recognized only, when they will actually practice midwifery and their practice can be made visible through media" (R02).

\section{Theme 2: Perspectives about Profession}

Facilitation of this programme largely depends upon the perception and qualities of a nurse who wants to become a midwife.

\section{Subtheme: Suitable Personal Qualities for a Midwife}

The attributes of the nurses to become an independent practitioner in midwifery is essential for this programme. Motivation to be a midwife is important as well as to show them the way or path to become equipped is a facilitator. The greatest deciding factor to promote midwifery is the midwife herself, her level of interest, commitment, competence; assertiveness plays a key role in acting as a facilitator.

"Independence is in the mind and in the skills, if the midwifes are skilled and knowledgeable nobody can stop them. Whether they can take decisions or not it is their mind. It all depends on how they promote their field and how much responsibility they can take and if they want, they can assert themselves and this is up to them how they feel good about themselves" (R09).

\section{Subtheme: Enrollment into the Profession by Choice}

The nurses who are going to be enrolled in this programme, needs to be monitored and recruited carefully with a set of certain criteria. The people who are passionate about the midwifery will definitely know about the importance of midwifery. The main facilitator at this point of time is that we have to enroll the passionate, skilled, competent nurses to become a practitioner in midwifery:

"We have to make sure that passionate midwives are enrolled for this programme" (R06).

"Experienced person and the new generation, those who are interested in midwifery should be enrolled" (R15).

\section{Theme 3: Educational Organization as Facilitators}

Since education is the powerful weapon for everything, educational organizations can act a catalyst towards initiation of the midwifery programme.

\section{Subtheme: Educational Endorsement}

Educators, who will educate these midwifes to become practitioners, have a vital role because their mode of teaching and their interest and ability to inculcate the professional qualities can act as a facilitator for this programme, so it is imperative that educational institutions providing this programme, have enough trained educators and this would entail training of educators.

"TOT- training of the trainers is imperative and the trainers should be carefully selected who are really dedicated to the midwifery profession, as they would further train the people with the same zest. Quality of training and education of the training of the trainer is vital" (R04).

"I think when there will be more established examples of midwifery and their success stories in the country, that will act as facilitators" (R18).

\section{Theme 4: Research Evidences}

The proved evidences on the midwifery care from other countries also will be one of the facilitators for this programme.

As we already have evidences that $85 \%$ of birth in India required only midwifery care so, it can further facilitate this programme to be implemented here, as we are already equipped with some background work done by the stakeholders will help into the initiation of this programme:

"Evidence that $85 \%$ of births can happen naturally and they do not require any medical intervention, which literally those $15 \%$ cases which can develop some complications" (R18).

\section{Theme 5: Enablers or Enabling Activities from Higher Authorities and Support from Other Countries}

To facilitate the midwifery programme, there are some activities from the authority which will be helpful for the initiation of this programme, as many key stakeholders addressed this in the account of the facilitators.

\section{Subtheme: Regulation}

Regulation or regulatory mechanism to control the profession or give recognition to the profession which is the best legal facilitator for the midwifery programme, which help the profession to acquaint with the success.

"There should be a separate Directorate of Nursing laid by a nursing personnel which should have horizontal linkages with the other programme" (R19).

"There should be proper guidelines; somebody should be monitoring all these things and feedback should be taken from the mothers as an evaluation of the services" (R02). 
Public private partnership also will be the facilitator, as we know we are somewhere lacking with the institutes in public sector so we have to do the partnership of both the public and private sector with the help of their administrators, so, this cooperation will further facilitate this programme:

\section{Subtheme: Technical Support}

Support from the other countries or within the country will facilitate this programme, as this point of time the support is very much important.

"The technical support like regular visits and engagement of international midwifery educator from countries which have implemented the midwifery programme is important" (R06).

\section{Theme 6: Professional Activities}

We are sending the midwife to the field to work for the mothers, so there is some facilitators at the work place also which will facilitate the programme to initiate: Working collaboratively with the doctors is also one of the facilitator for the programme:

\section{Subtheme: Professional Framework}

The model of care which the midwife follows will be a facilitator for this and teamwork with doctors and other members of the healthcare team is imperative, which will help the programme to initiate:

"The models which have been existing like models of care in Fernandez Hospital, India Bangladesh and UK can be taken as examples before actual implementation of this programme" (R04).

With the help of QDA Miner software it found that the major barrier perceived by the key stakeholders of midwifery were work place barrier (14.56\%) followed by educational barrier (13.25), individual barrier (12.38\%), barrier at policy level $(9.99 \%)$ and societal barrier was $6.84 \%$.

The facilitators perceived by the key stakeholders were enablers or enabling activities from higher authority and support from other countries (11.45\%) followed by prospective about profession (10.78\%), educational organization (8.14\%) and educational organization (6.77\%) sensitization of the society $(6.59 \%)$ and professional activities (6.56\%).

\section{Discussion}

The discussion is based on the themes that emerged as a result of the study that were developed on the basis of the research questions.

A stakeholder is an individual, group or organization who is impacted by the outcome of a project. They have an interest in the success of the project. In present study key stakeholders of midwifery were involved to explore their perceptions regarding the barriers to the initiation of midwifery programme which has been not initiated yet.

The present study's results showed that implementing the midwifery programme is a complex process that is influenced by variety of barriers. The study offers insight into the actual factors affecting the implementation of midwifery programme. Identifying these barriers can help set some priorities to overcome these barriers or lower these barriers which can help into the early initiation of midwifery programme in India.

The findings of the present study show that the barriers to the initiation of midwifery programme are perceptions associated with the lack of personal attributes and lack of adaptability of proficiency among nurses. This puts the midwifery programme in a vulnerable position to initiate. As the nurses those who are working in the maternal and child care units are not confident or competent enough to work in the field of midwifery. A similar study done by Thiessen $\mathrm{K}$ et al. in a North American country of Manitoba found that the society in which we live perceived the midwifery profession as antiquated. The attribution of these common stereotypes to midwifery appeared to have weakened the overall image of the profession. Ultimately, these stereotypes have impacted midwives' status negatively in other professional circles. ${ }^{6}$

In addition to this, the present study found that work place barriers prevent the initiation of midwifery programme which was reflected by the workplace environment, working style, recruitment issues and progression at workplace. Our findings put forward the concept that nurses in India are not utilized to their full capacity within the healthcare system. The reason for this is a lack of recognition and autonomy towards the nursing profession. Interestingly, a similar finding was presented in a study conducted by Bogren $\mathrm{M}$ et al. ${ }^{7}$ in Bangladesh that shows a vulnerable situation of midwives facing cultural prejudice and lack of proper security measures in the work place.

Another study conducted by Thiessen K et al. in North American country Manitoba found that the work environment as a factor contributing to burnout and high rates of attrition for the Manitoba midwives. ${ }^{6}$

In present study, societal barrier has been perceived to prevent midwifery programme to come into the practice in India, Midwives in India continue to face struggle to defend their professional status, which was reflected by the taking up of the profession with a business intention and for making profits from birth and the discrepancy in the opinion of public regarding the birth including the mother's bad experience during birth. This is not unique to India, as such low literacy rate and awareness towards the public is one which prevents this programme to come forward. Similar findings have been presented in a study 
conducted by Bogren M, Earland $\mathrm{K}$, et al. in Bangladesh, where people think midwives are young and unskilled in supporting a woman through childbirth and maternal and new born care. ${ }^{7}$

Midwives in India have lost control of their profession by not having effective midwifery educational programme. The lack of effective educational programme has been influenced by the lack of educational resources and institutes. Due to the less hours for midwifery in the course and the less trained or competent teachers, has overshadowed its importance to be a profession. Similar findings have been presented in a study conducted by Bogren $\mathrm{M}$ et al. in Bangladesh, where the government is struggling with the shortage of midwives as a barrier to quality care. Medical intern's doctors have dominated midwives' practice. $^{7}$

Findings show that in India there is some sailing force which pulls back the midwifery programme to be carried out. This includes the lack of regulatory support from the policy level. The Indian government does not make a strong policy decision to implement the midwifery programme.

The results of the present study highlighted that the sensitization among the society on midwifery by various measures can help in the initiation of this programme by means of creating visibility and awareness programmes. One of the stakeholders mentioned that the popular medias are showing bad pictures of childbirth in India, which we can be swapped with pleasurable childbirth pictures which can facilitate this programme. One of the similar studies in North American country Manitoba by Thiessen $\mathrm{K}$ et al. stated that women's strong desire for choice of birthplace and maternity care providers was the impetus to regulate midwifery in Manitoba and bring awareness to factors concerning the utilization of regulated midwifery services that have buried within structures of power. ${ }^{6}$

In the present study, it was found that, support from higher authority will help in the facilitation of this programme. If India has a regulation for midwives like separate council for midwifes which can give autonomy to the midwives along with the proper auditing, monitoring and evaluation system, it can definitely facilitate this programme. The study done in North America by Thiessen K et al. stated that most participants outlined various successes in the strategies to implement midwifery in Manitoba. For instance, a participant from the professional bodies commented on the fact that regulation was adequate since it included key components to support the profession. ${ }^{6}$

The key stakeholders in the present study stated that as of now India does not have an independent midwifery profession so, it will be useful if we learn from other countries and if they support us for its initiation this will be useful.
In our study key stakeholders of midwifery in New Delhi, India commented on professional activities which are desirable to be done at work place which can facilitate this programme, like team work at work place, model of care, scope of practice, legal framework at workplace.

\section{Conclusion}

It has been concluded from the study that it is vital to sensitize the community to have a natural birthing experience and instill a desire in them to receive a continuum of care by midwives. The study concludes that it is imperative to recruit trained faculties for imparting training coupled with adequate infrastructure, resources and facilities for training of midwives who will be enrolled for the midwifery programme. Policy makers have a key role to play to implement actions to overcome the barriers to the initiation of midwifery programme in India. This study can be a starting point as it collates the ideas, opinions and statements of key stakeholders of midwifery to address the burning issue of changing the status of midwifery profession in India.

\section{Acknowledgement}

We would like to express our gratitude towards all the participants who were taken their time from their busy schedule to contribute in this study and gave us the quality data for the study which is extremely helpful for the future as well. And Dr. Krishnamurthy Prabhakar, Professor, Head Research Initiatives, Alapakkam, Chennai-600049. He provided us with the assistant $\&$ support to organize and analyses the huge qualitative data in a representable form.

\section{Conflicts of Interest: None \\ References}

1. Birth \& its meanings: representations of birth in art. Available from: http://ecampusontario.pressbooks. pub/cmroleofmidwifery/chapter/births-and-itsmeaning/\#navigation.

2. Annual Report of Department of Health and Family Welfare 2017-18, Ministry of Health and Family Welfare.

3. India readies a new cadre of certified midwives to improve maternal, infant care. Available from: http:// www.indiaspend.com/india-readies-a-new-cadre-ofcertified-midwives-to-improve-maternal-infant-care/.

4. Strengthening quality midwifery education for Universal Health Coverage 2030. Available from: http://www. who.int/maternal_child_adolescent/topics/quality-ofcare/midwifery/strengthening-midwifery-education/ en/.

5. India has one doctor for every 1,457 citizens: Govt. Available from: Business-standard.com/article/ptistories/india-has-doctors-for-every-1-457-citizengovt-119070401127_1.html. 
6. Thiessen K, Heaman M, Mignone J et al. Barriers and facilitators related to implementation of regulated midwifery in Manitoba: A Case Study. BMC 2016; 16: 92.

7. Bogren $M$, Erlandsson $K$, Byrskog $U$ et al. What prevents midwifery quality care in Bangladesh? A focus group enquiry with midwifery students. BMC Health Services Research 2018; 18(1): 1-9. 